\title{
An Extended Value-Based Argumentation Framework for Ontology Mapping with Confidence Degrees
}

\author{
Cássia Trojahn ${ }^{1}$, Paulo Quaresma ${ }^{1}$ and Renata Vieira ${ }^{2}$ \\ ${ }^{1}$ Departamento de Informática, Universidade de Évora, Portugal \\ ${ }^{2}$ Pós-Graduacão em Computacão Aplicada, Universidade do Vale do Rio dos Sinos, \\ Brazil \\ cassia@di.uevora.pt,pq@di.uevora.pt,renatav@unisinos.br
}

\begin{abstract}
Heuristics to combine different approaches for ontology mapping have been proposed in the literature. This paper proposes to use abstract argumentation frameworks to combine such approaches. We extend the Value-based Argumentation Framework (VAF)[2], in order to represent arguments with confidence degrees. Our agents apply individual mapping algorithms and cooperate in order to exchange their local results (arguments). Next, based on their preferences and confidence of the arguments, the agents compute their preferred mapping sets. The arguments in such preferred sets are viewed as the set of globally acceptable arguments.
\end{abstract}

\section{Introduction}

Ontology mapping is the process of linking corresponding terms from different ontologies. The mapping result can be used for ontology merging, agent communication, query answering, or for navigation on the Semantic Web.

Well-known approaches to the problem can be grouped into lexical, semantic, and structural ones, as terms may be mapped by a measure of lexical similarity, or they can be evaluated semantically, usually on the basis of semantic oriented linguistic resources, or considering the term positions in the ontology hierarchy. It is assumed that the approaches are complementary to each other and combining different ones reflect better solutions when compared to the solutions of the individual approaches. Heuristics to combine such approaches have been proposed [18][14][9][15].

This paper proposes to use abstract argumentation frameworks [6] to combine approaches for ontology mapping. We extend a state of art argumentation framework, namely Value-based Argumentation Framework (VAF)[2], in order to represent arguments with confidence degrees. The VAF allows to determine which arguments are acceptable, with respect to the different audiences represented by different agents. We then associate to each argument a confidence degree, representing how confident an agent is in the similarity of two ontology terms. 
Our agents apply different mapping approaches and cooperate in order to exchange their local results (arguments). Next, based on their preferences and confidence of the arguments, the agents compute their preferred mapping sets. The arguments in such preferred sets are viewed as the set of globally acceptable arguments. Our approach is able to give a formal motivation for the composite mapping approaches.

This paper is structured as follows. Section 2 comments on argumentation framework. Section 3 introduces the ontology mapping approaches. Section 4 presents our agent argumentation model. Section 5 presents a walk through example. Section 6 comments on related work. Finally, section 7 presents the final remarks and the future work.

\section{Argumentation Framework}

Our argumentation model is based on the Value-based Argumentation Frameworks (VAF)[2], a development of the classical argument system of Dung [6]. First, we present the Dung's framework, upon which the VAF rely. Next, we present the VAF and our extended framework.

\subsection{Classical argumentation framework}

Dung [6] defines an argumentation framework as follows.

Definition 2.1.1 An Argumentation Framework is a pair $A F=(A R$, attacks $)$, where $A R$ is a set of arguments and attacks is a binary relation on AR, i.e., attacks $\subseteq \mathrm{AR} \times \mathrm{AR}$. An attack $(\mathrm{A}, \mathrm{B})$ means that the argument $\mathrm{A}$ attacks the argument B. A set of arguments $S$ attacks an argument B if B is attacked by an argument in $S$.

The key question about the framework is whether a given argument $A, A$ $\in A R$, should be accepted. One reasonable view is that an argument should be accepted only if every attack on it is rebutted by an accepted argument [6]. This notion produces the following definitions:

Definition 2.1.2 An argument $A \in A R$ is acceptable with respect to set arguments $S(\operatorname{acceptable}(A, S))$, if $(\forall x)(x \in A R) \&(\operatorname{attacks}(x, A)) \longrightarrow(\exists y)(y \in$ S) $छ \operatorname{attacks}(y, x)$

Definition 2.1.3 A set $S$ of arguments is conflict-free if $\neg(\exists x)(\exists y)((x \in S) \mathcal{G}(y$ $\in S) \& \operatorname{attacks}(x, y))$

Definition 2.1.4 A conflict-free set of arguments $S$ is admissible if $(\forall x)(x \in S)$ $\longrightarrow \operatorname{acceptable}(x, S)$

Definition 2.1.5 A set of arguments $S$ is a preferred extension if it is a maximal (with respect to inclusion set) admissible set of $A R$. 
A preferred extension represent a consistent position within $A F$, which can defend itself against all attacks and which cannot be further extended without introducing a conflict.

The purpose of [2] in extending the AF is to allow associate arguments with the social values they advance. Then, the attack of one argument on another is evaluated to say whether or not it succeeds by comparing the strengths of the values advanced by the arguments concerned.

\subsection{Value-based argumentation framework}

In Dung's frameworks, attacks always succeed. However, in many domains, including the one under consideration, arguments lack this coercive force: they provide reasons which may be more or less persuasive [11]. Moreover, their persuasiveness may vary according to their audience.

The VAF is able to distinguish attacks from successful attacks, those which defeat the attacked argument, with respect to an ordering on the values that are associated with the arguments. It allows accommodate different audiences with different interests and preferences.

Definition 2.2.1 A Value-based Argumentation Framework (VAF) is a 5-tuple $V A F=(A R$, attacks, $V$,val,$P)$ where $(A R$, attacks $)$ is an argumentation framework, $V$ is a nonempty set of values, val is a function which maps from elements of $A R$ to elements of $V$ and $P$ is a set of possible audiences. For each $A \in A R, \operatorname{val}(A) \in V$.

Definition 2.2.2 An Audience-specific Value Based Argumentation Framework (AVAF) is a 5-tuple $V A F_{a}=\left(A R\right.$, attacks, $V$, val,valpref $\left.f_{a}\right)$ where $A R$, attacks, $V$ and val are as for a VAF, $a$ is an audience and valpref $f_{a}$ is a preference relation (transitive, irreflexive and asymmetric) valpre $f_{a} \subseteq V \times V$, reflecting the value preferences of audience $a$. valpref $\left(v_{1}, v_{2}\right)$ means $v_{1}$ is preferred to $v_{2}$.

Definition 2.2.3 An argument $A \in A R$ defeats $_{a}$ (or successful attacks) an argument $B \in A R$ for audience $a$ if and only if both $\operatorname{attacks}(A, B)$ and not $\operatorname{valpref}(\operatorname{val}(B), \operatorname{val}(A))$.

An attack succeeds if both arguments relate to the same value, or if no preference value between the values has been defined.

Definition 2.2.4 An argument $A \in A R$ is acceptable to audience $a$ (acceptable a $\left._{a}\right)$ with respect to set of arguments $S$, acceptable act $\left._{(}(A, S)\right)$ if $(\forall x)((x \in A R \&$ defeats $\left._{a}(x, A)\right) \longrightarrow(\exists y)\left((y \in S) \&\right.$ defeats $\left.\left._{a}(y, x)\right)\right)$.

Definition 2.2.5 A set $S$ of arguments is conflict-free for audience $a$ if $(\forall x)(\forall$ $y)\left((x \in S \& y \in S) \longrightarrow\left(\neg \operatorname{attacks}(x, y) \vee \operatorname{valpref}(\operatorname{val}(y), \operatorname{val}(x)) \in \operatorname{valpref}_{a}\right)\right)$.

Definition 2.2.6 A conflict-free set of argument $S$ for audience $a$ is admissible for an audience $a$ if $(\forall x)\left(x \in S \longrightarrow\right.$ acceptable $\left._{a}(x, S)\right)$. 
Definition 2.2.7 A set of argument $S$ in the VAF is a preferred extension for audience $a$ (preferred ${ }_{a}$ ) if it is a maximal (with respect to set inclusion) admissible for audience $a$ of AR.

In order to determine the preferred extension with respect to a value ordering promoted by distinct audiences, [2] introduces the notion of objective and subjective acceptance.

Definition 2.2.8 An argument $x \in A R$ is subjectively acceptable if and only if $x$ appears in the preferred extension for some specific audiences but not all. An argument $x \in A R$ is objectively acceptable if and only if, $x$ appears in the preferred extension for every specific audience. An argument which is neither objectively nor subjectively acceptable is said to be indefensible.

\subsection{An extended value-based argumentation framework}

We extend the VAF in order to represent arguments with confidence degrees. Two elements have been added to the VAF: a set with confidence degrees and a function which maps from arguments to confidence degrees. The confidence value represents the confidence that an individual agent has in some argument. We assumed that the confidence degrees is a criteria which is necessary to represent the ontology mapping domain.

Definition 2.3.1 An Extended Value-based Argumentation Framework (E-VAF) is a 7 -tuple $E-V A F=(A R$, attacks, $V$,val, $P, C$, val $C)$ where $(A R$, attacks, $V$,val, $P)$ is a value-based argumentation framework, $C$ is a nonempty set of values representing the confidence degrees, valC is a function which maps from elements of $A R$ to elements of $C$. valC $\subseteq C \times C$ and valpref $C\left(c_{1}, c_{2}\right)$ means $c_{1}$ is preferred to $c_{2}$.

Definition 2.3.2 An argument $x \in A R$ defeats $_{a}$ (or successful attacks) an argument $y \in A R$ for audience $a$ if and only if $\operatorname{attacks}(x, y) \wedge(\operatorname{valprefC}(\operatorname{val} C(x)$, $\operatorname{val} C(y)) \vee(\neg \operatorname{valpref}(\operatorname{val}(y), \operatorname{val}(x)) \wedge \neg \operatorname{valpre} f C(\operatorname{val} C(y), \operatorname{val} C(x))))$.

An attack succeeds if (a) the confidence degree of the attacking argument is greater than the confidence degree of the argument being attacked; or if (b) the argument being attacked does not have greater preference value than attacking argument (or if both arguments relate to the same preference values) and the confidence degree of the argument being attacked is not greater than the attacking argument.

Definition 2.3.3 A set $S$ of arguments is conflict-free for audience $a$ if $(\forall x)(\forall y)$ $((x \in S \& \mathcal{E} y \in S) \longrightarrow(\neg \operatorname{attacks}(x, y) \vee(\neg$ valprefC $(\operatorname{val} C(x), \operatorname{val} C(y)) \wedge($ val$\operatorname{pref}(\operatorname{val}(y), \operatorname{val}(x)) \vee \operatorname{valpref} C(\operatorname{val} C(y), \operatorname{val} C(x)))))$. 


\section{Ontology Mapping}

The approaches for ontology mapping vary from lexical (see [18][14]) to semantic and structural levels (see [9]). In the lexical level, metrics to compare string similarity are adopted. One well-known measure is the Levenshtein distance or edit distance [12], which is given by the minimum number of operations (insertion, deletion, or substitution of a single character) needed to transform one string into another. Other common metrics can be found in [14], [17], and [7].

The semantic level considers the semantic relations between concepts to measure the similarity between them, usually on the basis of semantic oriented linguistic resources. The well-known WordNet $^{1}$ database, a large repository of English semantically related items, has been used to provide these relations. This kind of mapping is complementary to the pure string similarity metrics. It is common that string metrics yield high similarity between strings that represent completely different concepts (i.e, the words "score" and "store"). Moreover, semantic-structural approaches have been explored [3][9]. In this case, the positions of the terms in the ontology hierarchy are considered, i.e, terms more generals and terms more specifics are also considered as input to the mapping process.

Heuristics to combine different approaches for ontology mapping have been proposed in the literature. It is assumed that the approaches are complementary to each other and combining different ones reflect better solutions when compared to the solutions of the individual approaches.

We propose to use the E-VAF to combine such approaches. Our agents apply different mapping algorithms and cooperate in order to exchange their local results (arguments). Next, based on their preferences and confidence of the arguments, the agents compute their preferred mapping sets. The arguments in such preferred sets are viewed as the set of arguments globally acceptable (objectively or subjectively).

\section{E-VAF for Ontology Mapping}

In our model, dedicated agents encapsulate different mapping approaches. Each approach represents a different audience in an E-VAF, i.e, the agents' preferences are based on specific approach used by the agent. In this paper we consider three audiences: lexical (L), semantic (S), and structural (E) (i.e. $P=\{L, S, E\}$, where $P \in \mathrm{E}-\mathrm{VAF})$. We point out that our model is extensible to other audiences.

\subsection{Argumentation generation}

First, the agents work in an independent manner, applying the mapping approaches and generating mapping sets. The mapping result will consist of a set of all possible correspondences between terms of two ontologies. A mapping $m$

\footnotetext{
${ }^{1}$ http://www.wordnet.princeton.edu
} 
can be described as a 3 -tuple $m=\left(t_{1}, t_{2}, R\right)$, where $t_{1}$ corresponds to a term in the ontology $1, t_{2}$ corresponds to a term in the ontology 2 , and $R$ is the mapping relation resulting from the mapping between these two terms. The lexical and semantic agents are able to return equivalence value to $R$, while the structural agents return sub-class or super-class values to $R$. Each mapping $m$ is represented as a argument. Now, we can define arguments as follows:

Definition 4.1 An argument $\in A R$ is a 4-tuple $x=(m, a, c, h)$, where $m$ is a mapping; $a \in P$ is the agent's audience generating that argument (agent's preference, i.e, lexical, semantic or structural); $c \in C$ is the confidence degree associated to that mapping (certainty or uncertainty, as it will be commented below); $h$ is one of $\{-,+\}$ depending on whether the argument is that $m$ does or does not hold.

The confidence degree is defined by the agent when applying the specific mapping approach. Here, we assumed $C=\{$ certainty, uncertainty $\}$, where $C \in$ E-VAF. Table 1 shows the possible values to $h$ and $c$, according to the agent's audiences. The agents generate their arguments based on rules from Table 1.

Table 1. $h$ and $c$ to audiences.

\begin{tabular}{|c|c|c|c|}
\hline & & \multicolumn{2}{|c|}{ Audiences } \\
\hline$h$ & $c$ & Lexical & Semantic \\
\hline+ & certainty & 1 & synonym \\
+ & uncertainty & $1>\mathrm{r}>\mathrm{t}$ & related \\
- & certainty & $0<\mathrm{r}<=\mathrm{t}$ & \\
- & uncertainty & 0 & unknown \\
\hline
\end{tabular}

Lexical agent The output of lexical agents $(r)$ is a value from the interval $[0,1]$, where 1 indicates high similarity between two terms. This way, if the output is 1 , the lexical agent generates an argument $x=(\mathrm{m}, \mathrm{L}$, certainty, +$)$, where $m=$ $\left(\mathrm{t}_{1}, \mathrm{t}_{2}\right.$, equivalence). If the output is 0 , the agent generates an argument $x=$ (m,L,certainty,-), where $m=\left(\mathrm{t}_{1}, \mathrm{t}_{2}\right.$, equivalence $)$. A threshold $(t)$ is used to classify the output in uncertain categories. The threshold value can be specified by the user.

Semantic agent The semantic agents consider semantic relations between terms, such as synonym, antonym, holonym, meronym, hyponym, and hypernym (i.e., such as in WordNet database). When the terms being mapped are synonymous, the agent generates an argument $x=(\mathrm{m}, \mathrm{S}$, certainty, +$)$, where $m=\left(\mathrm{t}_{1}, \mathrm{t}_{2}\right.$, equivalence $)$. The terms related by holonym, meronym, hyponym, or hypernym are considered related and an argument $x=(\mathrm{m}, \mathrm{S}$, uncertainty, +$)$ is generated, where $m=\left(\mathrm{t}_{1}, \mathrm{t}_{2}\right.$, equivalence $)$; when the terms can not be related by 
the WordNet (the terms are unknown for the WordNet database), an argument $x=(\mathrm{m}, \mathrm{L}$, uncertainty, -$)$, where $m=\left(\mathrm{t}_{1}, \mathrm{t}_{2}\right.$, equivalence $)$, is then generated.

Structural agent The structural agents consider the super-classes (or subclasses) intuition to verify if the terms can be mapped. First, it is verified if the super-classes of the compared terms are lexically similar. If not, the semantic similarity between they is used. If the super-classes of the terms are lexically or semantically similar, the terms are considered equivalent to each other. The argument is generated according to the lexical or semantic comparison. For instance, if the super-classes of the terms are not lexically similar, but they are synonymous (semantic similarity), an argument $x=(\mathrm{m}, \mathrm{E}$, certainty,+$)$, where $m=\left(\mathrm{t}_{1}, \mathrm{t}_{2}\right.$, super-class $)$, is generated.

\subsection{Preferred extension generation}

After generating their set of arguments, the agents exchange with each other their arguments. Following a specific protocol, an agent asks (ask sign) the others about their arguments. The other agents then, send their arguments to the first agent. An ack sign is then sent to requesting agents, in order to indicate that the arguments have been correctly received. Otherwise, an error sign is sent.

When all agents have received the set of argument of the each other, they generate their attacks set. An attack (or counter-argument) will arise when we have arguments for the mapping between the same terms, but with conflicting values of $h$. For instance, an argument $x=\left(\mathrm{m}_{1}, \mathrm{~L}\right.$, certainty, +$)$ have as an attack an argument $y=\left(\mathrm{m}_{2}, \mathrm{E}\right.$, certainty,- $)$, where $\mathrm{m}_{1}$ and $\mathrm{m}_{2}$ refer to the same terms in the ontologies. The argument $y$ also represents an attack to the argument $x$.

As an example, consider the mapping between the terms "Reference/ Dissertation" and "Citation/Thesis" and the lexical and structural agents. The lexical agent generates an argument $x=(\mathrm{m}, \mathrm{L}$, uncertainty, $)$, where $m=$ (dissertation,thesis,equivalence); and the structural agent generates an argument $y=$ $(\mathrm{m}, \mathrm{E}$, certainty,+$)$, where $m=$ (dissertation,thesis, super-class). For both lexical and structural audiences, the set of arguments is $A R=\{\mathrm{x}, \mathrm{y}\}$ and the attacks $=$ $\{(\mathrm{x}, \mathrm{y}),(\mathrm{y}, \mathrm{x})\}$. However, the relations of successful attacks will be defined according to specific audience (see Definition 2.3.2), as it is commented below.

When the set of arguments and attacks have been produced, the agents need to define which of them must be accepted. To do this, the agents compute their preferred extension, according to the audiences and confidence degrees. A set of arguments is globally subjectively acceptable if each element appears in the preferred extension for some agent. A set of arguments is globally objectively acceptable if each element appears in the preferred extension for every agent. The arguments which are neither objectively nor subjectively acceptable are considered indefensible.

In the example above, considering the lexical(L) and structural(E) audiences, where $\mathrm{L} \succ \mathrm{E}$ and $\mathrm{E} \succ \mathrm{L}$, respectively. For the lexical audience, the argument $y$ successful attacks the argument $x$, while the argument $x$ does not successful 
attack the argument $y$ for the structural audience. Then, the preferred extension of both lexical and structural agents is composed by the argument $y$, which can be seen as globally objectively acceptable. The mapping between the terms "Reference/ Dissertation" and "Citation/Thesis", indicated by $y$ is correct.

\section{A walk through example}

Let us consider that three agents need to obtain a consensus about mappings that link corresponding class names in two different ontologies.

First, we used part of the ontology of Google and Yahoo web directories ${ }^{2}$, and the argumentation model output has been compared with manual matches ${ }^{3}$.

We considered lexical (L), semantic (S), and structural (E) audiences (mapping approaches) in order to verify the behavior of our argumentation model. The lexical agent was implemented using the edit distance measure (Levenshtein measure). We used the algorithm available in the API for ontology alignment $(\text { INRIA })^{4}$ (EditDistNameAlignment). The semantic agent has used the JWordNet $\mathrm{API}^{5}$, which is an interface to the WordNet database. For each WordNet synset, we retrieved the synonymous terms and considered the hypernym, hyponym, member-holonym, member-meronym, part-holonym, and part-meronym as related terms. The structural agent was based on super-classes similarity. The threshold used to classify the matcher agents output was 0.6 . This value was defined based on previous analysis of the edit distance values between the terms of the ontologies used in the experiments. The terms with edit distance values greater than 0.6 have presented lexical similarity.

We have selected three possible mappings between terms of the ontologies: "Music/History" and "Architecture/History", "Art/ArtHistory" and "ArtHumanity/ArtHistory", and "Art" and "ArtHumanity". Table 2 shows arguments and attacks (counter-arguments) generated for each audience. The mappings between these terms have been selected because they were identified as conflicting cases when using our previous cooperative negotiation model [20][21], which is based on voting mechanism.

For the mapping between the terms "Music/History" and "Architecture/ History", each agent has as arguments $A R=\{1,2,3\}$ and as relations of attack attacks $=\{(3,1),(3,2),(1,3),(2,3)\}$. These sets are generated by each agent, after receiving the arguments of other agents. Next, the arguments that defeat each other are computed, according to the agent's audience. For the lexical audience, where $\mathrm{L} \succ \mathrm{S}$ and $\mathrm{L} \succ \mathrm{E}$, there is no argument that successfully attacks another, because all agents have certainty in the mappings. The same occurs for the semantic $(\mathrm{S} \succ \mathrm{L}$ and $\mathrm{S} \succ \mathrm{E}$ ) and structural $(\mathrm{E} \succ \mathrm{L}$ and $\mathrm{E} \succ \mathrm{S})$ audiences.

The preferred extensions of the agents are composed by the arguments generated by the corresponding audience (i.e, the preferred extension of the lexical

\footnotetext{
${ }^{2}$ http://dit.unitn.it/ãccord/Experimentaldesign.html (Test 3)

${ }^{3}$ http://dit.unitn.it/ accord/Experimentaldesign.html

${ }^{4}$ http://alignapi.gforce.inria.fr

${ }^{5}$ http://jwn.sourceforge.net (using WordNet 2.1)
} 
Table 2. Arguments and attacks.

\begin{tabular}{|c|c|c|}
\hline & Argument & Attacks \\
\hline 1 & (history,history, equivalence,L, certainty,+) & 3 \\
\hline 2 & (history,history, equivalence, $\mathrm{S}$, certainty, + ) & 3 \\
\hline 3 & (history,history, super-class,E,certainty,-) & 1,2 \\
\hline 4 & $($ (art-history,art-history, equivalence,L, certainty,+) & - \\
\hline 5 & (art-history,art-history, equivalence, $\mathrm{S}$, certainty,+$)$ & \\
\hline 6 & (art-history,art-history,super-class,E, certainty,+) & \\
\hline 7 & (art,art-humanity,L, equivalence, uncertainty,-) & 8,9 \\
\hline 8 & (art,art-humanity,S, equivalence, certainty,+) & 7 \\
\hline 9 & (art,art-humanity,E,super-class, uncertainty,+) & 7 \\
\hline
\end{tabular}

agent is $\{1\}$; the preferred extension of the semantic agent is $\{2\}$; and the preferred extension of the structural agent is $\{3\}$ ). This way, there is no argument globally objectively acceptable. Then, we can consider that the mapping between the terms is not possible, what is correct according to the manual mapping.

Using our cooperative negotiation model, the final mapping between the "Music/History" and "Architecture/ History" terms was incorrect. The semantic and lexical agents returned mappings with certainty, while the structural agent returned a non mapping with certainty. By voting, a mapping with certainty was obtained. This conflict is then resolved by our argumentation model.

For the mapping between the terms "Art/ArtHistory" and "ArtHumanity/ArtHistory", each agent has as arguments $A R=\{4,5,6\}$, but there are not relations of attack. Then, all agents accept the mapping with certainty between these terms. This mapping is considered a correct mapping by the manual mapping.

Finally, for the mapping between the terms "Art" and "ArtHumanity", each agent has as arguments $A R=\{7,8,9\}$ and as relations of attack attacks $=\{(8,7)$, $(9,7),(7,8),(7,9)\}$. For the lexical audience, the argument 8 successfully attacks the argument 7 . Then, the preferred extension has the argument 8 . For the semantic audience, the argument 8 also successful attacks the argument 7 , and for structural audience, the arguments 8 and 9 successful attack theirs counterarguments. Then, the preferred extension of the structural agent is $\{8,9\}$. The argument 8 is present in all preferred extension, then it is globally objectively acceptable, confirming the mapping indicated by manual mapping.

We have used different agents' output which use distinct mapping algorithms in order to verify the behavior of our model. Our argumentation model has identified correctly the three mappings defined by expert mappings, being two mapping positives $(h$ is +$)$ and one negative $(h$ is -$)$.

Second, we compared the argumentation output with the results obtained by the cooperative negotiation model. Table 3 shows the comparative results. Although the negotiation model having obtained better precision than argumentation model, the F-measure of the argumentation model is better than negotiation model. The negotiation model identified 7 true positive mappings and it did not 
classify correctly 4 true positive mappings. The argumentation model identified 8 true positive, returning 1 false positive mapping not identifying 3 true positives mappings.

Table 3. Argumentation vs. negotiation.

\begin{tabular}{|l||c|c|c|c|c|c|}
\hline \multicolumn{1}{|c||}{ Ontology } & \multicolumn{3}{|c|}{ Argumentation } & \multicolumn{3}{|c|}{ Negotiation } \\
\hline Company profiles (160) & 0.88 & 0.72 & 0.79 & 1 & 0.63 & 0.77 \\
\hline \hline
\end{tabular}

Third, we compared our argumentation model with three state of the art schema-based matching systems: Cupid [10], COMA [5], and S-Match [8]. We consider the class and the attribute names of the ontologies in the comparison. Table 4 shows the results. Our argumentation model had better F-measures than all others systems.

Table 4. Comparative mapping results - argumentation model.

\begin{tabular}{|l||c|c|c|c|c|c|c|c|c|c|c|c|}
\hline \multicolumn{1}{|c||}{} & \multicolumn{3}{c|}{ Arg } & \multicolumn{3}{c|}{ Cupid } & \multicolumn{3}{c|}{ COMA } & \multicolumn{3}{c|}{ S-Match } \\
Ontology & $\mathrm{P}$ & $\mathrm{R}$ & $\mathrm{F}$ & $\mathrm{P}$ & $\mathrm{R}$ & $\mathrm{F}$ & $\mathrm{P}$ & $\mathrm{R}$ & $\mathrm{F}$ & $\mathrm{P}$ & $\mathrm{R}$ & $\mathrm{F}$ \\
\hline \hline Company profiles (160) & 0.88 & 0.72 & 0.79 & 0.50 & 0.60 & 0.54 & 0.80 & 0.70 & 0.74 & 1.0 & 0.65 & 0.78 \\
\hline
\end{tabular}

\section{Related Work}

In the field of ontology negotiation we find distinct proposals. [19] presents an ontology to serve as the basis for agent negotiation, the ontology itself is not the object being negotiated. A similar approach is proposed by [4], where ontologies are integrated to support the communication among heterogeneous agents.

[1] presents an ontology negotiation model which aims to arrive at a common ontology which the agents can use in their particular interaction. We, on the other hand, are concerned with delivering mapping pairs found by a group of agents using abstract argumentation frameworks. The links between related concepts are the result of the preferred mappings of each agent, instead of an integrated ontology upon which the agents will be able to communicate for a specific purpose. We do not consider negotiation steps such as the ones presented in [1], namely clarification and explanation. But we consider different mapping methods represented by different audiences selecting by argumentation the best solution for the mapping problem.

[16] describes an approach for ontology mapping negotiation, where the mapping is composed by a set of semantic bridges and their inter-relations, as proposed in [13]. The agents are able to achieve a consensus about the mapping 
through the evaluation of a confidence value that is obtained by utility functions. According to the confidence value the mapping rule is accepted, rejected or negotiated. Differently from [16], we do not use utility functions. Our model is based on cooperation and argumentation, where the agents exchange their arguments and by argumentation they select the preferred mapping. The arguments in each preferred set are considered globally acceptable.

[11] proposes to use an argument framework to deal with arguments that support or oppose candidate correspondences between ontologies. The mapping candidates are provided by a single service. The accepted mappings resulting from argumentation are used for agent communication. Differently from [11], the mappings are obtained by different agents specialized on different mapping algorithms and not only in a single service. In [11], the mappings are assumed to be correct, and we are interested in how to obtain mapping sets by combining different approaches for ontology mapping. Moreover, in [11] it is assumed that arguments being negotiated have the same confidence. We are proposing to associate to each argument a confidence degree. This way, in order to compute the preferred mapping, the audiences and confidence degrees must be considered.

\section{Final Remarks and Future Work}

This paper proposed to use abstract argumentation frameworks to combine approaches for ontology mapping. We extended a state of art argumentation framework, namely Value-based Argumentation Framework (VAF), in order to represent arguments with confidence degrees. The VAF allows to determine which arguments are acceptable, with respect to the different preferences represented by different agents. Our extension associates to each argument a confidence degree, representing the confidence that a specific agent has in that argument. We assumed that the confidence degrees is a criteria which is necessary to represent the ontology mapping domain.

We have used different agents' output which use distinct mapping algorithms in order to verify the behavior of our model. The terms presented here were identified as conflicting cases in our previous negotiation model. Our argumentation model has identified correctly the three mapping defined by expert mappings, being two mapping positives ( $h$ is + ) and one negative ( $h$ is -). This model has obtained better results for the conflicting cases when compared with our previous model. When comparing our model with the three state of the art matching systems, our model obtained better F-measure than all other systems. The results, although preliminary, are promising especially for what concerns F-measure values.

In the future, we intend to develop further tests considering also agents using constraint-based mapping approaches; and use the ontology's application context in our matching approach. Next, we will use the mapping result as input to an ontology merge process in the question answering domain. 


\section{Acknowledgments}

The first author is supported by the Programme Alban, the European Union Programme of High Level Scholarships for Latin America, scholarship no.E05D059374BR.

\section{References}

1. S. Bailin and W. Truszkowski. Ontology negotiation between intelligent information agents. The Knowledge Engineering Review, 17(1):7-19, 2002.

2. T. Bench-Capon. Persuasion in practical argument using value-based argumentation frameworks. Journal of Logic and Computation, 13:429-448, 2003.

3. M. Chaves. Mapeamento e comparacao de similaridade entre estruturas ontologicas. Master's thesis, Pontificia Universidade Catolica do Rio Grande do Sul, 2002.

4. J. v. Diggelen, R. Beun, F. Dignum, v. R. Eijk, and J. C. Meyer. Anemone: An effective minimal ontology negotiation environment. In Proceedings of the $V$ International Conference on Autonomous Agents and Multi-Agent Systems, pages 899-906, 2006.

5. H. H. Do and E. Rahm. Coma - a system for flexible combination of schema matching approaches. In Proceedings of the 28th Conference on Very Large Databases (VLDB), 2002.

6. P. Dung. On the acceptability of arguments and its fundamental role in nonmonotonic reasoning, logic programming and n-person games. Artificial Intelligence, 77:321-358, 1995.

7. J. Euzenat, T. Le Bach, J. Barrasa, P. Bouquet, J. De Bo, R. Dieng-Kuntz, M. Ehrig, M. Hauswirth, M. Jarrar, R. Lara, D. Maynard, A. Napoli, G. Stamou, H. Stuckenschmidt, P. Shvaiko, S. Tessaris, S. Van Acker, and I. Zaihrayeu. State of the art on ontology alignment. Technical report, 2004.

8. F. Giunchiglia, P. Shvaiko, and M. Yatskevich. S-match: An algorithm and an implementation of semantic matching. In First European Semantic Web Symposium, 2004.

9. F. Hakimpour and A. Geppert. Resolving semantic heterogeneity in schema integration: an ontology approach. In Proceedings of the International Conference on Formal Ontology in Informational Systems, 2001.

10. P. B. J. Madhavan and E. Rahm. Generic schema matching with cupid. In Proceedings of the Very Large Data Bases Conference (VLDB), page 49, 2001.

11. L. Laera, V. Tamma, J. Euzenat, T. Bench-Capon, and T. R. Payne. Reaching agreement over ontology alignments. In Proceedings of 5th International Semantic Web Conference (ISWC 2006).

12. I. Levenshtein. Binary codes capable of correcting deletions, insertions an reversals. In Cybernetics and Control Theory, 1966.

13. A. Maedche, B. Motik, N. Silva, and R. Volz. Mafra - a mapping framework for distributed ontologies. In 13th International Conference on Knowledge Engineering and Knowledge Management, pages 235-250, 2002.

14. A. Maedche and S. Staab. Measuring similarity between ontologies. In Proceedings of the European Conference on Knowledge Acquisition and Management, pages 251-263, 2002.

15. A. Rodriguez and M. Egenhofer. Determining semantic similarity among entity classes from different ontologies. IEEE Transactions on Knowledge and Data Engineering, 15(2):442. 
16. N. Silva, P. Maio, and J. Rocha. An approach to ontology mapping negotiation. In Proceedings of the K-CAP Workshop on Integrating Ontologies.

17. T. Smith and M. Waterman. Identification of common molecular subsequences. Journal of Molecular Biology, 147:195-197, 1981.

18. G. Stoilos, G. Stamou, and S. Kollias. A string metric for ontology alignment. In ISWC, pages 624-637. 4th International Semantic Web Conference (ISWC 2005), 2005.

19. V. Tamma, M. Wooldridge, I. Blacoe, and I. Dickinson. An ontology based approach to automated negotiation. In Proceedings of the IV Workshop on Agent Mediated Electronic Commerce, pages 219-237, 2002.

20. C. Trojahn, M. Moraes, P. Quaresma, and R. Vieira. A negotiation model for ontology mapping. In Proceedings of the IEEE/WIC/ACM International Conference on Intelligent Agent Technology, 2006.

21. C. Trojahn, M. Moraes, P. Quaresma, and R. Vieira. Using cooperative agent negotiation for ontology mapping. In Proceedings of Fourth European Workshop on Multi-Agent Systems (EUMAS), 2006.

This article was processed using the IATEX macro package with LLNCS style 